\title{
THE EQUIVALENCE OF SEIBERG-WITTEN AND CASSON INVARIANTS FOR HOMOLOGY 3-SPHERES
}

\author{
YUHAN LIM
}

\begin{abstract}
We outline a proof that the Seiberg-Witten invariant for integral homology 3-spheres is the same as Casson's invariant. This confirms a conjecture of Kronheimer.
\end{abstract}

\section{Introduction}

In 1985, Casson [AM] introduced an integer invariant $\lambda$ for oriented integral homology 3 -spheres. He determined the change of the invariant under the effect of Dehn surgery. Let $Y$ be a homology 3 -sphere and $K$ a knot in $Y$. Let $Y_{ \pm 1}$ denote the manifold obtained by \pm 1 -surgery on $K$. Then Casson obtained:

$$
\lambda\left(S^{3}\right)=0, \quad \lambda\left(Y_{ \pm 1}\right)=\lambda(Y) \pm \frac{1}{2} \Delta_{K}^{\prime \prime}(1),
$$

where $\Delta_{K}(t)$ is the symmetrized Alexander polynomial of $K$, normalized so that $\Delta_{K}(1)=1$. In fact these two relations completely determine $\lambda$.

On the other hand the recently introduced Seiberg-Witten equations define invariants for oriented 3-manifolds which in the case of homology spheres is an integer valued invariant, which we shall denote by $\tau$. (For an exposition see [L1].) It was conjectured by P. Kronheimer that these two invariants are in fact the same. This conjecture was based on the verification of the equality of the two invariants for the class of Brieskorn homology spheres based on computations in [MOY]. It is the aim of this article to answer this conjecture in the affirmative.

Theorem A. The Seiberg-Witten invariant for oriented integral homology 3spheres is equal to the Casson invariant.

To prove this it will suffice to show that $\tau$ satisfies (1). To establish such a surgery formula we will apply the methods developed in [L2] to relate the moduli spaces for the surgered and unsurgered manifolds. The additional complication in the case at hand is the necessity of introducing certain metric dependent counter terms in the definition of $\tau$; these are spectral invariants of [APS]. (We shall review the definition of $\tau$ below.) A final ingredient in the proof is to identify certain terms involving $\tau$ for manifolds with $b_{1}=1$. This requires the Theorem of Meng-Taubes [MT]. The mod 2 version of Theorem A is also proven in $[\mathrm{Ch}]$.

Received March 11, 1997. Revised September 8, 1999. 
Acknowledgement. The author would like to thank Alan Carey for the generous hospitality at the University of Adelaide during which this work was completed.

\section{Preliminaries}

SW invariants. We briefly recall some of the definitions of the invariants, in the general case, without the assumption of a homology sphere. Unless otherwise stated, we will work in the $C^{\infty}$ category and $C^{\infty}$ topologies. Now $Y$ denotes an oriented closed 3-manifold. For SW we will also require an auxiliary Riemannian metric on $Y$. Let $P \rightarrow Y$ denote a spin-c structure, $L(P)$ denote the associated canonical determinant line bundle, and $S(P)$ the spinor bundle. We shall always choose $S(P)$ to be the one for which Clifford action by the volume form is multiplication by +1 . Let $\omega$ denote a closed pure-imaginary 1-form and $\alpha$ a pure-imaginary 1-form. Then for a pair $(A, \Phi)$ of a connection on $L(P)$ and section of $S(P)$ we have the perturbed SW-equations:

$$
F_{A}=\sigma(\Phi, \Phi)+\omega, \quad D_{A} \Phi+\alpha \cdot \Phi=0 .
$$

The gauge group is the space of maps $Y \rightarrow S^{1} \subset \mathbf{C}$ and its action on $(A, \Phi)$ is by $\left(A+2 g^{-1} d g, g^{-1} \Phi\right)$.

The moduli space of solutions is denoted by $Z_{\alpha, \omega}(P)$. If the irreducible part $Z_{\alpha, \omega}^{*}(P)$ of the moduli space is finite and regular, then each point is canonically oriented and we may form the algebraic sum $\# Z_{\alpha, \omega}^{*}(P)$.

Theorem 1. There exists arbitarily small perturbations of $(\alpha, \omega)$ (we call these generic) such that the following is true. If $b_{1}(Y)>1, Z_{\alpha, \omega}(P)$ is a finite set of canonically oriented irreducible regular points; furthermore $\# Z_{\alpha, \omega}(P)$ is independent of the choice of $(\alpha, \omega)$ and metric on $Y$. If $b_{1}(Y)=1$ then same is also true provided $\omega$ is small in homology (see below). If $b_{1}(Y)=0$ then $Z_{\alpha, \omega}(P)$ is a finite set as above but with one reducible point. $\# Z_{\alpha, \omega}^{*}(P)$ is independent of choice of $(\alpha, \omega)$ and metric after addition of an appropriate counter term which is a combination of spectral invariants of Atiyah-Patodi-Singer.

The counter term for an integral homology sphere $Y$ with metric $g$ is defined as follows (see [L1] for more details). Let $\eta(D, g)$ be the value at 0 of the $\eta$ invariant for the canonically defined Dirac operator $D$ on $Y$ (i.e., that which is coupled to the trivial connection) with respect to the metric $g$. We also have a spectral invariant $\eta(d, g)$ which is the $\eta$ invariant at 0 for $* d-d *$ on even forms on $Y$. Assume first the special situation of a 'good' metric $g$ on $Y$, i.e., one for which $D$ is invertible. Then for any perturbation $(\alpha, \omega)$ sufficiently small, the counter-term takes the form

$$
c(Y, g)=\frac{1}{8} \eta(d, g)+\frac{1}{2} \eta(D, g) .
$$


The fact that this is an integer is a consequence of the alternative expression for $c(Y, g)$ as:

$$
-\left(\frac{1}{8} \operatorname{signature}(X)+\operatorname{Index}_{L^{2}} D(\widehat{X}, h)\right)
$$

where $X$ is a compact oriented spin 4-manifold with boundary $Y$ and $D(\widehat{X}, h)$ refers to the Dirac operator on the manifold $\widehat{X}=X \cup(\partial X \times[0, \infty))$ equipped with a metric $h$ which is a product $[0, \infty) \times(Y, g)$ on the end $\partial X \times[0, \infty) . c(Y, g)$ is constant over all sufficiently small changes in $g$.

Then the following combination is $\mathbb{Z}$-valued and is a topological invariant: (since $Y$ has a unique spin-c structure we do not have to specify it)

$$
\# Z_{\alpha, \omega}^{*}(Y)+c(Y, g)
$$

where $(\alpha, \omega)$ is small and generic in the sense of Theorem 1. This defines $\tau$ for integral homology spheres.

If $D$ is not invertible then, as in [L1], start with an arbitarily small $\alpha_{0}$ so that $D+\alpha_{0}$. is invertible. Then for all $(\alpha, \omega)$ sufficiency close to $\left(\alpha_{0}, 0\right)$, the previously defined counter-term is modified by the addition of an extra term $\frac{1}{32 \pi^{2}} \int_{Y} a \wedge \alpha$ where $d a=\alpha$. Denote the counter-term in this case by $c\left(Y, g, \alpha_{0}\right)$. A similiar equivalent expression for $c\left(Y, g, \alpha_{0}\right)$, as before, shows that it is also an integer and constant for all sufficiently small changes in $g$ and all $(\alpha, \omega)$ close to $\left(\alpha_{0}, 0\right)$. A topological invariant is defined just as before but this time with $Z_{\alpha+\alpha_{0}, \omega}^{*}(Y)$ replacing $Z_{\alpha, \omega}^{*}(Y)$.

For later purposes we also discuss the invariant for manifolds with $b_{1}=1$. The (perturbed) moduli space admits a reducible solution if and only if $\omega$ and $F_{A}$ are cohomologous. Thus if $\omega$ is varying and passes through the de Rham class of $F_{A}$ the moduli space develops singularities and thus $\# Z_{\alpha, \omega}(P)$ may change. To formalize this, let $\left[\frac{i}{2 \pi} \omega\right]$ be the de Rham class of $\frac{i}{2 \pi} \omega$ in $H^{2}(Y ; \mathbb{R})$. Then for generic $(\alpha, \omega)$ the value of $\# Z_{\alpha, \omega}(P)$ depends on the component of $H^{2}(Y ; \mathbb{R})-c_{1}(L(P))$ to which $\left[\frac{i}{2 \pi} \omega\right]$ belongs. This component is called the chamber of $\omega$. The change as we vary from one chamber to another is given in [L1] (note that because all oriented 3-manifolds are spin, $c_{1}(L(P)$ ) which is represented by $\frac{i}{2 \pi} F_{A}$ is always divisible by 2 in $H^{2}(Y ; \mathbb{Z}) /$ torsion):

Theorem 2. If $b_{1}(Y)=1$, then $\# Z_{\alpha, \omega}(P)$ is an oriented diffeomorphism invariant of $Y$ depending only on a choice of chamber for $\omega$ in $H^{2}(Y ; \mathbb{R})$. Let $\left[\omega_{0}\right] \in H^{2}(Y ; \mathbb{Z}) /$ torsion be indivisible and $\frac{1}{2} c_{1}(L(P))=n\left[\omega_{0}\right]$ modulo torsion. Then as $\omega$ changes from the chamber $(n-1 / 2)\left[\omega_{0}\right]$ to the chamber $(n+1 / 2)\left[\omega_{0}\right]$, $\# Z_{\alpha, \omega}(P)$ changes by $n$. In particular $\# Z_{\alpha, \omega}(P)$ is a well-defined invariant if $\omega$ is sufficiently small.

For manifolds with $b_{1}=1$ we define $\tau(P)$ to be $\# Z_{\alpha, \omega}(P)$ where $\omega$ is sufficiently small in homology and generic. Here small means the evaluation of $\omega$ over any primitive integral homology class is small. 
Cylindrical-End Manifolds. We summarise some results from [L2]. Let $Y$ be an oriented Riemannian 3-manifold with cylindrical-end $T^{2} \times[0, \infty)$ where $T^{2}$ is a 2-torus, and thus has a flat metric. Then for a compatible cylindrical-end spinc structure $P \rightarrow Y$ we may consider a moduli-space $Z_{\alpha, \omega}(P)$ of $\mathrm{SW}$-solutions exponentially decaying to a flat connection and vanishing spinor field [L2]. In particular we shall always work with $(\alpha, \omega)$ which have compact support, say in $Y-T^{2} \times(0, \infty){ }^{1}$

In a point which will play a role later, note that the gauge group action $A \mapsto A+2 g^{-1} d g$ is by 'even' transformations (that is, they have even degree as maps to $S^{1}$ ), for these are the only ones that lift to automorphisms of the spin-c structure. For example not all the trivial connections need be actually equivalent in this way.

We need some additional notation: $\chi_{e}$ denotes the space of flat $U(1)$ connections on $T^{2}$ modulo even gauge transformations. We have a natural projection map $R: Z_{\alpha, \omega}(P) \rightarrow \chi_{e}$ which takes each point to its asymptotic value. The structure of $R^{-1}([\varrho])$ depends heavily on the linearized equations at infinity, at the flat connection $\varrho$. This linearization is the Dirac operator on $T^{2}$ coupled to $\varrho$. A simple argument shows there is a unique (even) gauge equivalence class $\Lambda$ of flat connection such that the coupled Dirac operator on the spinor bundle with trivial canonical class has non-trivial kernel. Analytically this is regarded as a non-smooth point, even though $\chi_{e}$ itself is just a 2-torus again.

We shall work away from this singular point: let $\epsilon>0$ be small and $U_{\epsilon}$ the complement in $\chi_{e}$ of a closed $\epsilon$-neighbourhood of $\Lambda$.

Here is a version of the structure theorem proved in [L2]:

Theorem 3. Let $Y$ be a cylindrical-end manifold as above with $H_{1}(Y ; \mathbb{Z}) \cong \mathbb{Z}$. Fix $\epsilon>0$ small so that $R\left(Z_{0}^{\text {Red }}\right) \subset U_{\epsilon}$. There are arbitarily small perturbations $(\alpha, \omega)$ with compact support (call these generic) such that the following holds. Denote $R^{-1}\left(U_{\epsilon}\right)$ by $Z_{\alpha, \omega}\left(U_{\epsilon}\right)$.

1. The reducible portion $Z_{\alpha, \omega}^{\operatorname{Red}}(P) \cong S^{1}$ and $Z_{\alpha, \omega}^{\operatorname{Red}}(P) \subset R^{-1}\left(U_{\epsilon}\right)$.

2. $\overline{Z_{\alpha, \omega}^{*}\left(U_{\epsilon}\right)}$ forms a smooth compact 1-manifold with boundary. The boundary points are reducible or lie in $R^{-1}\left(\partial \bar{U}_{\epsilon}\right)$.

3. Let $x \in \overline{Z_{\alpha, \omega}\left(U_{\epsilon}\right)} \cap Z_{\alpha, \omega}^{\text {Red }}(P)$. Then a neighbourhood of $x$ is modeled on the zeros of the map $\mathbb{R} \times \mathbb{R}^{+} \rightarrow \mathbb{R},(t, z) \rightarrow t z$ with $\mathbb{R} \times\{0\}$ corrsponding to the reducibles.

4. The asymptotic value map $R$ is smooth on $Z_{\alpha, \omega}^{*}\left(U_{\epsilon}\right)$.

5. $\left.R\right|_{Z_{\alpha, \omega}\left(U_{\epsilon}\right)}$ is transverse to any given finite subcomplex of $\chi_{e}$.

6. $Z_{\alpha, \omega}^{*}\left(U_{\epsilon}\right)$ and $Z_{\alpha, \omega}^{\mathrm{Red}}(P)$ are both oriented by an orientation of $H^{1}(Y ; i \mathbb{R})$ (see below for details).

Since the reducibles will play an important role later we collect some observations here. If $\omega=0$ we can explicitly write down the map $R^{\text {Red }}$, the restriction of $R$ on the reducibles since these are just flat connections up to even-gauge.

\footnotetext{
${ }^{1}$ This is different from the moduli space considered by Meng-Taubes.
} 
This is denoted by $\chi_{e}(Y) . R^{\text {Red }}$ is then the natural restriction map

$$
R^{\mathrm{Red}}: \chi_{e}(Y) \rightarrow \chi_{e}
$$

induced by the inclusion $T^{2} \rightarrow Y-T^{2} \times[0, \infty)$. With a small perturbation $\omega$ present then $Z_{\alpha, \omega}^{\operatorname{Red}}(Y) \cong \chi_{e}(Y)$ and with this identification the image of $R_{\alpha, \omega}^{\text {Red }}$ is the image of a function of the form $R^{\text {Red }}+f(\omega)$, where addition in $\chi_{e}$ is interpreted in the universal cover (an affine space modelled on $H^{1}\left(T^{2} ; i \mathbb{R}\right)$ ).

Let us examine a point of $\overline{Z_{\alpha, \omega}\left(\chi_{e}^{0}\right)} \cap Z_{\alpha, \omega}^{\text {Red }}$ carefully (assuming generic $(\alpha, \omega)$ ). Locally such a point looks like a ' $\mathrm{T}$ ' with the horizontal arm the reducibles. The singular point $\left(A_{0}, 0\right)$ is characterised by the Dirac operator $D_{A_{0}}$ (on $L^{2}$-sections) coupled to $A_{0}$ having non-trivial (in fact complex dimension 1 ) kernel. Genericity means that the family $D_{A}$ as $A$ varies over the reducibles has transverse spectral flow at $A_{0}$. ( $D_{A}$ is self-adjoint as an operator on $L^{2}$ and the notion of spectral flow in this context is defined - see [CLM].) Let us suppose an orientation of $H^{1}(Y ; \mathbb{R})$ is given. The orientation on the irreducibles near $\left(A_{0}, 0\right)$ either points into or away from the reducibles. This is entirely determined by the sign of the spectral flow of $D_{A}$ at the singular point as $A$ moves through the reducibles ([L2]). The tangent space to the reducibles is identified with $H^{1}(Y ; i \mathbb{R})$ and therefore is oriented by $H^{1}(Y ; i \mathbb{R})$. If the spectral flow at $A_{0}$ is positive (moving in the direction of the orientation on the reducibles) then the orientation on the irreducibles is into $\left(A_{0}, 0\right)$. The opposite situation occurs when the spectral flow is negative.

Now suppose $Y$ is a compact oriented Riemannian 3-manifold and $Y=Y_{+}^{\prime} \cup_{T^{2}}$ $Y_{-}^{\prime}$ with $H_{1}\left(Y_{ \pm}^{\prime} ; \mathbb{Z}\right) \cong \mathbb{Z}$. Denote by $Y_{T}$ the Riemannian manifold obtained by fitting a cylinder $T^{2} \times[-T, T]$ between $Y_{+}^{\prime}$ and $Y_{-}^{\prime}$. As $T \rightarrow \infty$ we have the geometric limits the cylindrical-end manifolds $Y_{ \pm}=Y_{ \pm} \cup_{T^{2}} T^{2} \times[0, \infty)$. Let $P_{ \pm} \rightarrow Y_{ \pm}$be given compatible cylindrical-end spin-c structures. Then gluing arguments give:

Theorem 4. Let $P_{ \pm} \rightarrow Y_{ \pm}, Y_{T}$, etc., be as above. Denote by $P_{T} \rightarrow Y_{T}$ a spin-c structure formed by fitting together $P_{ \pm} \rightarrow Y_{ \pm}$. Assume $Z_{0}^{*}\left(P_{-}\right)=\emptyset$. Let $\epsilon>0$ be sufficiently small so that $R_{+}\left(Z_{0}^{\operatorname{Red}}\left(P_{+}\right)\right) \cup R_{-}\left(Z_{0}\left(P_{-}\right)\right) \subset U_{\epsilon}$. Choose a small generic perturbation $(\alpha, \omega)$ so that Theorem 3 holds for $Z_{\alpha, \omega}\left(P_{+}\right)$ with $\left.R_{+}\right|_{Z_{\alpha, \omega}\left(U_{\epsilon}\right)}$ transverse to $R_{-}$. Then for $T$ sufficiently large there is an orientation preserving diffeomorphism

$$
\Upsilon_{T}: Z_{\alpha, \omega}\left(U_{\epsilon}\right) \times_{\chi_{e}} Z_{0}^{\mathrm{Red}}\left(P_{-}\right) \rightarrow \bigcup_{P_{T}} Z_{\alpha, \omega}\left(P_{T}\right)
$$

where the union is taken over isomorphism classes of spin-c structures $P_{T}$. Furthermore each $Z_{\alpha, \omega}\left(P_{T}\right)$ satisfies the conclusion of Theorem 1. Given any constant compact set $K \subset Y_{T}$ the $C^{k}$ distance between $(x, y)$ and $\Upsilon_{T}(x, y)$ over $K$ is of $o(1)$ as $T \rightarrow \infty$. 


\section{Proof of Theorem A}

Let $Y$ denote an oriented integral homology 3 -sphere and $K$ a knot in $Y$. Let $\nu K=D^{2} \times S^{1}$ be a (closed) tubular neighbourhood, so we may write $Y=(Y-\nu K) \cup D^{2} \times S^{1}$. Let $m, l$ denote a standard meridian-longitude pair on $T^{2}=\partial(Y-\nu K)$. This means the following. $m$ is the boundary of an embedded disk in $\nu K$, and $m$ is given an orientation. $l$ is the oriented boundary of the portion of a Seifert surface $\Sigma$ for $K$ in $Y-(\nu K)^{\circ}$ such that:

(i) $l$ is an embedded curve in $\partial \nu K$,

(ii) $l$ meets $m$ in exactly one point,

(iii) the intersection number of $m$ and $\Sigma$ is +1 in $Y$.

We choose a metric on $Y$ satisfing the the following:

1. The metric on $T^{2}$ is the standard product metric $S^{1} \times S^{1}$.

2. The curve $S^{1} \times\{1\}$ is homologous to $m$.

3. The curve $\{1\} \times S^{1}$ is homologous to $m+l$.

4. The metric on $D^{2} \times S^{1}$ is the product metric of the standard +1 -scalar curvature metric on $D^{2}$ (as the upper hemisphere of $S^{2}$ ) and $S^{1}$.

With this set-up +1 -surgery (the argument for $(-1)$-surgery is symmetric and thus omitted here) on $K$ is achieved by simply changing the identification along $T^{2}$ by an isometry $h: \partial(Y-\nu K) \rightarrow \partial\left(D^{2} \times S^{1}\right)$ which interchanges $S^{1} \times\{1\}$ and $\{1\} \times S^{1}$ (and thus keeping the positive scalar curvature condition on $D^{2} \times S^{1}$ ). Denote this manifold by $Y_{1}$. For 0 -surgery we can change the identification by a map $h^{\prime}$; but this is no longer an isometry of $T^{2}$ with the given metric. $h^{\prime}$ pushes forward the standard metric to a flat metric on $\partial\left(D^{2} \times S^{1}\right)$. After some thought the reader will see that we can interpolate between two flat metrics on $T^{2} \times[0,1]$ such that the result has non-negative scalar curvature. Therefore we assume non-negative scalar curvature metric on $D^{2} \times S^{1}$ which gives us 0 -surgery. We fix once and for all this metric which we denote by $g_{0}$. Denote the 0 -surgered manifold by $Y_{0}$.

As in the usual gauge theory arguments, we insert a tube $T^{2} \times[-T, T]$ to obtain cylindrical-neck manifolds $Y_{T}, Y_{1, T}$ and $Y_{0, T}$. Letting $T \rightarrow \infty$ we obtain as geometric limits the cylindrical-end manifolds $\hat{Y}=(Y-\nu K) \cup_{T^{2}} T^{2} \times[0, \infty)$ and $\widehat{W}, \widehat{W}^{\prime}=D^{2} \times S^{1} \cup_{T^{2}} T^{2} \times[0, \infty)$ where $\widehat{W}$ corresponds to the product metric on $D^{2} \times S^{1}$ and $\widehat{W}^{\prime}$ the non-product metric $g_{0}$. Since we have essentially unique spin-c structures on $\widehat{Y}, \widehat{W}$ and $\widehat{W}^{\prime}$ we shall not introduce notation for them.

In our set-up there there will be positive scalar curvature on $\widehat{W}$ and nonnegative on $\widehat{W}^{\prime}$ and thus by the scalar curvature bound property for the SeibergWitten equations there will be no irreducible solutions (to the unperturbed equations). Thus $Z_{0}(\widehat{W})=\chi_{e}(\widehat{W})=S^{1}$ and similiarly for $\widehat{W}^{\prime}$.

Choose $\epsilon>0$ small, $(\alpha, \omega)$ small and generic, so that Theorem 4 applies with $Y_{+}=\widehat{Y}$ and $Y_{-}=\widehat{W}$ or $\widehat{W}^{\prime}$. Then for $T$ large the irreducible part of the $(\alpha, \omega)$-perturbed moduli space for the unique spin-c structure on $Y_{T}, Y_{1, T}$, 
and the union over all spin-c strucures for $Y_{0, T}$, is given by the intersection of $R\left(Z_{\alpha, \omega}^{*}\left(U_{\epsilon}\right)\right)$ with $R\left(\chi_{e}(\widehat{W})\right), R_{1}\left(\chi_{e}(\widehat{W})\right), R_{0}\left(\chi_{e}\left(\widehat{W}^{\prime}\right)\right)$ respectively in $\chi_{e}$. Here $R_{1}, R_{0}$ is $R$ followed by the map induced on $\chi_{e}$ by $h, h^{\prime}$ respectively. In particular the above mentioned intersections are all transverse. Given the above we shall drop specifying spin-c structures in further notation.

Let us introduce coordinates on $\chi_{e}$. On the determinant of the (unique) spinc structure on $\widehat{Y}$ the 'trivial' connections (i.e., with trivial holonomy) fall into exactly two gauge equivalence classes, let us write these as $[\theta],\left[\theta^{\prime}\right]$. We can distinguish $[\theta]$ to be the gauge class which 'extends' over $Y_{T}$. Using the image of $[\theta]$ under $R$, which we also denote as $[\theta]$, as a basepoint the coordinates $x, y$ on $\chi_{e}$ are obtained by taking the integral over $m, l$ respectively of $A-\theta$ modulo $4 \pi i$ (assume $S^{1} \subset \mathbf{C}$ is of unit radius). Then

1. $R\left(Z_{\alpha, \omega}^{\operatorname{Red}}(\widehat{Y})\right)$ is represented by the line $y=\epsilon^{\prime}$ where $\left|\epsilon^{\prime}\right| \neq 0$ is small, depending on $\omega$.

2. $[\theta]=(0,0),\left[\theta^{\prime}\right]=(2 \pi i, 0)$.

3. $\Lambda$ is the point $(2 \pi i, 2 \pi i)$.

4. $R\left(\chi_{e}(\widehat{W})\right)$ is the $y$-axis.

5. $R_{1}\left(\chi_{e}(\widehat{W})\right)$ is the line $x+y+2 \pi i=0$.

6. $R_{0}\left(\chi_{e}\left(\widehat{W}^{\prime}\right)\right)$ is the $x$-axis.

7. $R\left(\overline{Z_{\alpha, \omega}^{*}}\left(U_{\epsilon}\right)\right)$ is a finite number of closed arcs with end-points either on $y=\epsilon^{\prime}$ (singular points) or on the circle of radius $\epsilon$ centered at $(2 \pi i, 2 \pi i)$.

At this point we need to mention the issue of orientations. The meridian $m$ as a class in $H_{1}(Y-\nu K ; \mathbb{Z})$ determines an orientation $0 \neq i \alpha_{Y} \in H^{1}(\widehat{Y} ; i \mathbb{R}) \cong i \mathbb{R}$ by the rule $\int_{m} \alpha_{Y}>0$. Therefore $Z_{\alpha, \omega}^{*}\left(U_{\epsilon}\right)$ and $Z_{\alpha, \omega}^{\text {Red }}(\widehat{Y})$ are both oriented. Define classes $\alpha$ and $\beta$ in $H^{1}\left(T^{2} ; \mathbb{R}\right)$ by $\int_{m} \alpha=1, \int_{l} \alpha=0, \int_{m} \beta=0$, and $\int_{l} \beta=1$. Since the tangent space to $\chi_{e}$ is $H^{1}\left(T^{2} ; i \mathbb{R}\right)$, an orientation of $H^{1}\left(T^{2} ; i \mathbb{R}\right)$ gives an orientation for $\chi_{e}$. Now choose the orientation $(i \alpha) \wedge(i \beta)$ for $H^{1}\left(T^{2} ; i \mathbb{R}\right)$ and therefore $\chi_{e}$. In our coordinates $x, y$ above this corresponds to $\partial_{x} \wedge \partial_{y}$. According to our conventions (see Appendix), an orientation for $\chi_{e}(\widehat{W}), \chi_{e}\left(\widehat{W}^{\prime}\right)$ is uniquely determined by orientations on $H^{1}(Y ; i \mathbb{R})$ and $H^{1}\left(T^{2} ; i \mathbb{R}\right)$. These work out to be:

1. For $Y_{T}, R\left(\chi_{e}(\widehat{W})\right)$ is oriented in direction $\partial_{y}$.

2. For $Y_{1, T}, R_{1}\left(\chi_{e}(\widehat{W})\right)$ is oriented in direction $-\partial_{x}+\partial_{y}$.

3. For $Y_{0, T}, R_{0}\left(\chi_{e}\left(\widehat{W}^{\prime}\right)\right)$ is oriented in direction $-\partial_{x}$.

Lastly our conventions dictate that $\# Z_{\alpha, \omega}^{*}\left(Y_{T}\right), \# Z_{\alpha, \omega}^{*}\left(Y_{1, T}\right), \# Z_{\alpha, \omega}^{*}\left(Y_{0, T}\right)$ is given by the intersection number

$$
R\left(Z_{\alpha, \omega}^{*}\left(U_{\epsilon}\right)\right) \cdot R\left(\chi_{e}(\widehat{W}), R\left(Z_{\alpha, \omega}^{*}\left(U_{\epsilon}\right)\right) \cdot R_{1}\left(\chi_{e}(\widehat{W}), R\left(Z_{\alpha, \omega}^{*}\left(U_{\epsilon}\right)\right) \cdot R_{0}\left(\chi_{e}\left(\widehat{W}^{\prime}\right)\right.\right.\right.
$$

respectively, taking note that intersection product is taken in the given order. In all intersection products, unless specifically mentioned, we make the convention of taking $R\left(Z_{\alpha, \omega}^{*}\left(U_{\epsilon}\right)\right)$ first. 
To reduce notational congestion and to simplify the arguments, assume a metric such that the Dirac operators $D_{\theta}$ and $D_{\theta^{\prime}}$ on $\widehat{Y}$ have trivial $L^{2}$-kernel. (In the general situation we work with a perturbation of the of the form $D_{A}+\alpha_{0}$., where $\alpha_{0}$ has compact support in $Y-(\nu K)$ and makes the operator non-singular for $A=\theta, \theta^{\prime}$ (see [L2]). A non-zero $\alpha_{0}$ necessitates usage of the more general counter-term $c\left(Y, g, \alpha_{0}\right)$ in defining the $\mathrm{SW}$-invariant below; but the additional term $\int_{Y} a \wedge \alpha_{0}$ is always supported in $Y-(\nu K)$ and the same arguments will be seen to go thru.)

Define $\gamma(t)=\theta+t\left(\theta^{\prime}-\theta\right), 0 \leq t \leq 1$ to be the path of flat connections on $\widehat{Y}$ joining $\theta$ to $\theta^{\prime}$. [ $[\gamma]$ is oriented consistently with the orientation of $Z_{\alpha, \omega}^{\operatorname{Red}}(\widehat{Y})$ in the sense that if $\omega \rightarrow 0$, then the two orientations coincide. $R([\gamma])$ is, in our model above, the portion of the $x$-axis given by $0 \leq x \leq 2 \pi i$ and oriented in the $\partial_{x}$ direction.

Proposition 1. For T sufficiently large the following holds:

$$
\# Z_{\alpha, \omega}^{*}\left(Y_{1, T}\right)=\# Z_{\alpha, \omega}^{*}\left(Y_{T}\right)+\# Z_{\alpha, \omega}\left(Y_{0, T}\right)-\mathrm{SF}\left(D_{A} ; \gamma\right)
$$

where $\operatorname{SF}\left(D_{A} ; \gamma\right)$ denotes the spectral flow on $\widehat{Y}$ from $\theta$ to $\theta^{\prime}$ along $\gamma$.

Proof. With the given orientations, we can deform the curve represented by $R_{1}\left(\chi_{e}(\widehat{W})\right)$ throught a family of isotopic curves $R(\epsilon), 0 \leq \epsilon \leq 1$ with the final curve $(\epsilon=1)$ the union of $R\left(\chi_{e}(\widehat{W})\right)$ and $y=\epsilon^{\prime}$ (this last curve is clearly isotopic to $\left.R_{0}\left(\chi_{e}\left(\widehat{W}^{\prime}\right)\right)\right)$. This deformation can be chosen so that for each $\epsilon \neq 1, R(\epsilon)$ meets $y=\epsilon^{\prime}$ only at $\left(2 \pi i(1-\epsilon), \epsilon^{\prime}\right)$. As $\left(2 \pi i(1-\epsilon), \epsilon^{\prime}\right)$ passes through a singular point, the intersection number $R\left(Z_{\alpha, \omega}^{*}(\widehat{Y})\right) \cdot R(\epsilon)$ changes by the negative of the spectral flow at that singular point. Now if $\omega$ is choosen to be sufficiently small so that $\left|\epsilon^{\prime}\right|$ is also small then the spectral flow from the reducible represented by $\left(0, \epsilon^{\prime}\right)$ to the reducible $\left(2 \pi i, \epsilon^{\prime}\right)$ (with respect to the orientation on $Z_{\alpha, \omega}^{\operatorname{Red}}(\widehat{Y})$ ) is equal to the spectral flow along $\gamma$. From this the claimed formula is easily seen.

We now discuss the counter terms for $\tau\left(Y_{1, T}\right)$ and $\tau\left(Y_{T}\right)$.

Proposition 2. There exists a constant $C$ depending only on $W, W^{\prime}$ so that for $T$ sufficiently large the following holds.

$$
c\left(Y_{1, T}\right)=c\left(Y_{T}\right)+\mathrm{SF}\left(D_{A} ; \gamma\right)+C .
$$

Proof. We are simply changing $Y_{1, T}$ to $Y_{T}$ by cutting open along a slice in the cylindrical neck and regluing by an isometry. For $\eta(d, g)$ this changes by a fixed amount, as worked out in $[\mathrm{MR}]$, in fact by $1^{2}$. That is, we have

$$
\eta\left(d, g_{1, T}\right)-\eta\left(d, g_{0, T}\right)=1 .
$$

(The precise value of the constant will not matter at this point.)

The term $\eta(D ; g)$ needs to be treated carefully. First we note that the canonical Dirac operators (i.e., coupled to the trivial connection, unique up to even

\footnotetext{
${ }^{2}$ This can also be seen by considering the surgery cobordism.
} 
gauge equivalence) on $Y_{1, T}$ and $Y_{T}$ do not agree on $\widehat{Y}-T^{2} \times[T, \infty)$ nor on $\widehat{W}-T^{2} \times[T, \infty)$. Over $\widehat{Y}-T^{2} \times[T, \infty)$ it restricts to $D_{\theta}$, whereas for $Y_{1, T}$ it restricts to $D_{\theta^{\prime}}$. If we define $\bar{\theta}$ and $\bar{\theta}^{\prime}$ on $\widehat{W}$ just as we did for $\widehat{Y}$ then the canonical Dirac operators can be formed by coupling to the spliced connections over $Y_{1, T}$ and $Y_{T}: \theta \#_{T} \bar{\theta}$ and $\theta^{\prime} \#_{T} \bar{\theta}^{\prime}$. By this we mean that $\theta \#_{T} \bar{\theta}$ is a trivial connection which agrees with $\theta$ over $\widehat{Y}-T^{2} \times[0, \infty)$ and with $\widehat{W}-T^{2} \times[0, \infty)$, and similiarly for $\theta^{\prime} \#_{T} \bar{\theta}^{\prime}$. We may therefore write the Dirac operators as $D_{\theta \#_{T} \bar{\theta}}$, $D_{\theta^{\prime} \#_{T} \bar{\theta}^{\prime}}$ respectively. We shall need to compare these two operators.

We may extend $\theta$ and $\theta^{\prime}$ on $\widehat{Y}-T^{2} \times[T, \infty)$ over $Y_{0, T}$ as trivial connections on the trivial determinant: we write these as $\theta \#_{T} \hat{\theta}$ and $\theta^{\prime} \#_{T} \hat{\theta}^{\prime}$ where $\hat{\theta}$ and $\hat{\theta}^{\prime}$ are now over $\widehat{W}^{\prime}$ (similiar comments for $\theta \#_{T} \hat{\theta}, \theta^{\prime} \#_{T} \hat{\theta}^{\prime}$ hold as in the previous paragraph). Let $\gamma^{\prime}$ have the same definition as the previously defined path $\gamma$, but applied to $\widehat{W}^{\prime}$. Then in a manner analogous to forming $\theta \#_{T} \hat{\theta}$, etc., we can consider the path of flat connections $\gamma \#_{T} \gamma^{\prime}$ on $Y_{0, T}$. This is a path joining $\theta \#_{T} \hat{\theta}$ to $\theta^{\prime} \#_{T} \hat{\theta}^{\prime}$. Denote the metric on $Y_{1, T}, Y_{T}, Y_{0, T}$ by $g_{1, T}, g_{T}, g_{0, T}$ respectively. According to $[\mathrm{APS}]$, the $\xi$-invariant for a flat connection $\varrho$

$$
\xi(\varrho)=\frac{1}{2}\left(\operatorname{dim} \operatorname{ker} D_{\varrho}+\eta\left(D_{\varrho}\right),\right)
$$

is constant as $\varrho$ varies except for integer jumps equal to the spectral flow of $D_{\varrho}$. Thus

$$
\frac{1}{2} \eta\left(\theta \#_{T} \hat{\theta} ; g_{0, T}\right)-\frac{1}{2} \eta\left(\theta^{\prime} \#_{T} \hat{\theta}^{\prime} ; g_{0, T}\right)=\operatorname{SF}\left(D_{A} ; \gamma \#_{T} \gamma^{\prime}\right)
$$

There exist $\eta$ invariants (evaluated at 0 ) for the Dirac operators $D_{B}, B=$ $\theta, \theta^{\prime}, \bar{\theta}$, etc., acting on $L^{2}$-sections on our cylindrical-end manifolds ([MM]). For us, two conditions hold:

1. The tangential operator on $T^{2}$ for the Dirac operator $D_{B}$ is invertible i.e., on the end $T^{2} \times[0, \infty), D_{B}$ is of the form $\frac{\partial}{\partial t}+D^{\prime}$ where $D^{\prime}$ is invertible.

2. The Dirac operator $D_{B}$ on $L^{2}$-sections is invertible.

Then according to $[\mathrm{MM}]$ we have the following relations as $T \rightarrow \infty$ :

$$
\begin{aligned}
\eta\left(D_{\theta \#_{T} \hat{\theta}} ; g_{0, T}\right) & =\eta\left(D_{\theta}\right)+\eta\left(D_{\hat{\theta}}\right)+o(1), \\
\eta\left(D_{\theta^{\prime} \#_{T} \hat{\theta}^{\prime}} ; g_{0, T}\right) & =\eta\left(D_{\theta^{\prime}}\right)+\eta\left(D_{\hat{\theta}^{\prime}}\right)+o(1), \\
\eta\left(D_{\theta \#_{T} \bar{\theta}} ; g_{1, T}\right) & =\eta\left(D_{\theta}\right)+\eta\left(D_{\bar{\theta}}\right)+o(1), \\
\eta\left(D_{\theta^{\prime} \#_{T} \bar{\theta}^{\prime}} ; g_{T}\right) & =\eta\left(D_{\theta^{\prime}}\right)+\eta\left(D_{\bar{\theta}^{\prime}}\right)+o(1) .
\end{aligned}
$$

This gives us a formula of the form

$$
\eta\left(D_{\theta \#_{T} \bar{\theta}} ; g_{1, T}\right)-\eta\left(D_{\theta^{\prime} \#_{T} \bar{\theta}^{\prime}} ; g_{T}\right)=\eta\left(D_{\theta \#_{T} \hat{\theta}} ; g_{0, T}\right)-\eta\left(D_{\theta^{\prime} \#_{T} \hat{\theta}^{\prime}} ; g_{0, T}\right)+C+o(1)
$$

where $C$ depends only on ( $W$, standard metric) and $\left(W^{\prime}, g_{0}\right)$. Finally we also have for $T$ large the relation $[\mathrm{CLM}]$

$$
\operatorname{SF}\left(D_{A} ; \gamma \#_{T} \gamma^{\prime}\right)=\operatorname{SF}\left(D_{A} ; \gamma\right)+\operatorname{SF}\left(D_{A} ; \gamma^{\prime}\right) \text {. }
$$


Since $\gamma^{\prime}(t)$ is flat and $\widehat{W}^{\prime}$ has non-negative scalar curvature a Weitzenbock argument implies there are no $L^{2}$-solutions for $D_{\gamma^{\prime}(t)}$ and so the term $\operatorname{SF}\left(D_{A} ; \gamma^{\prime}\right)=0$. Hence from (3), (4) and (5) we obtain

$$
\frac{1}{2} \eta\left(D_{\theta \#_{T} \bar{\theta}} ; g_{1, T}\right)-\frac{1}{2} \eta\left(D_{\theta^{\prime} \#_{T} \bar{\theta}^{\prime}} ; g_{T}\right)=\mathrm{SF}\left(D_{A} ; \gamma\right)+\frac{1}{2} C+o(1) .
$$

Combined with (2) we obtain

$$
c\left(Y_{1, T}\right)-c\left(Y_{T}\right)=\operatorname{SF}\left(D_{A} ; \gamma\right)+\frac{1}{2} C+o(1) .
$$

For large $T, D_{\theta \#_{T} \bar{\theta}}$ and $D_{\theta^{\prime} \#_{T} \bar{\theta}^{\prime}}$ are invertible and therefore the left hand sum of (6) is independent of $T$ since it depends only on the spectral flow of these operators (as a function of $T$ ). Hence the $o(1)$ terms vanish for $T$ large.

Corollary 1. There is a universal constant $C$ so that the following holds:

$$
\tau\left(Y_{1}\right)=\tau(Y)+\sum_{P} \tau\left(Y_{0}\right)(P)+C .
$$

Our next task is to identify the term $\sum_{P} \tau\left(Y_{0}\right)(P)$.

Proposition 3. Let $\Delta_{K}(t)$ be the symmetrized Alexander Polynomial of $K$ and normalized so that $\Delta_{K}(1)=1$. Then

$$
\sum_{P} \tau\left(Y_{0}\right)(P)=\frac{1}{2} \Delta_{K}^{\prime \prime}(1)
$$

Proof. Fix a generator $a$ for $H^{2}\left(Y_{0} ; \mathbb{Z}\right)$ and let $P_{n}$ be the spin-c structure whose first chern class is $2 n a$. Then we can form the series

$$
\tau(t)=\sum \tau\left(P_{n}\right) t^{n}
$$

Here we are assuming $\omega$ is small. Then we wish to evaluate this at $t=1$. Now by the wall-crossing formula Theorem 2 , as we let $\omega \rightarrow \infty$ we pick-up the formal series

$$
t+2 t^{2}+3 t^{3}+\cdots=\frac{t}{(1-t)^{2}} .
$$

But according to Meng-Taubes $[\mathrm{MT}]$, for $\omega \rightarrow \infty$, we have

$$
\tau_{\infty}(t)=\frac{\Delta_{K}(t)}{\left(t^{1 / 2}-t^{-1 / 2}\right)^{2}}=\frac{t \Delta_{K}(t)}{(1-t)^{2}}
$$

Thus in the case of $\omega$ small we have

$$
\tau(t)=\frac{t\left(\Delta_{K}(t)-1\right)}{(1-t)^{2}} .
$$

Now an easy computation identifies $\tau(1)$ with $\frac{1}{2} \Delta_{K}^{\prime \prime}(1)$. Note that in this calculation we need to use the fact that $\Delta_{K}^{\prime}(1)=0$ (cf., $[\mathrm{AM}]$ ).

To complete the proof of Theorem A we just observe that the constant $C$ in Corollary 1 must be zero, by considering $S^{3}$ and the unknot, and $\tau\left(S^{3}\right)=0$. 


\section{Appendix}

We briefly indicate the necessary steps to establish the orientations for $R\left(\chi_{e}(\widehat{W})\right), R_{1}\left(\chi_{e}(\widehat{W})\right)$ and $R_{0}\left(\chi_{e}\left(\widehat{W}^{\prime}\right)\right)$ in the proof of Proposition 1. We have $Z_{\alpha, \omega}^{*}\left(U_{\epsilon}\right)$ a smooth 1-dimensional manifold. Over $Z_{\alpha, \omega}^{*}\left(U_{\epsilon}\right)$ we have a determinant line $\Xi_{Y}$ which as mentioned in Theorem 3 is oriented by an orientation of $H^{1}(\widehat{Y} ; i \mathbb{R})$. More precisely, $\Xi_{\widehat{Y}}$ is canonically isomorphic (up to multiplication by a positive scalar in the fibres) to the constant line $H^{1}(\widehat{Y} ; i \mathbb{R})$. Over $\chi_{e}(\widehat{W})$, $\chi_{e}\left(\widehat{W}^{\prime}\right)$ the determinant line is exactly $H^{1}(\widehat{W} ; i \mathbb{R}), H^{1}\left(\widehat{W}^{\prime} ; i \mathbb{R}\right)$ respectively and this is precisely the tangent space itself.

Now let $\Xi_{Y_{*}}\left(Y_{*}=Y_{T}, Y_{1, T}\right.$ or $\left.Y_{0, T}\right)$ denote the determinant line which orients the corresponding moduli space. Then over the image of the gluing map $\Upsilon_{T}$ of Theorem 4 we have the relation (due to excision)

$$
\Xi_{Y_{*}} \cong \Xi_{Y} \otimes H^{1}\left(W^{\dagger} ; i \mathbb{R}\right) \otimes \Lambda^{2} H^{1}\left(T^{2} ; i \mathbb{R}\right)^{*},
$$

where $W^{\dagger}=\widehat{W}$ or $\widehat{W}^{\prime}$. The $H^{1}\left(T^{2} ; i \mathbb{R}\right)$ term should be regarded as the tangent space to $\chi_{e}$. As shown in [L1], $\Xi_{Y_{*}}$ is canonically isomorphic (up to multiplication by a positive scalar in the fibres) to the trivial constant line $H^{1}\left(Y_{*} ; i \mathbb{R}\right) \otimes H^{2}\left(Y_{*} ; i \mathbb{R}\right)^{*}$ (apply Poincare duality). On the other hand at a regular point $x$ of the moduli space, $\Xi_{Y_{*}}$ is canonically trivialized with $i \mathbb{R}$. Comparison of the this with the trivialization given by $H^{1}\left(Y_{*} ; i \mathbb{R}\right) \otimes H^{2}\left(Y_{*} ; i \mathbb{R}\right)^{*}$ determines the \pm 1 -orientation of $x$. We make contact with the orientation of $\Xi_{Y}$ by the relation

$$
H^{1}\left(Y_{*} ; i \mathbb{R}\right) \otimes H^{2}\left(Y_{*} ; i \mathbb{R}\right)^{*} \cong H^{1}(\widehat{Y} ; i \mathbb{R}) \otimes H^{1}\left(W^{\dagger} ; i \mathbb{R}\right) \otimes \Lambda^{2} H^{1}\left(T^{2} ; i \mathbb{R}\right)^{*}
$$

Comparison of (7) and (8) establishes the orientation of a point $\Upsilon_{T}(a, b)$ as the intersection number between $R\left(Z_{\alpha, \omega}^{*}\left(U_{\epsilon}\right)\right)$ and $R\left(\chi_{e}(\widehat{W})\right)$, etc., at $\Upsilon_{T}(a, b)$.

The isomorphism (8) is simply a manifestation of the Mayer-Vietoris sequence relating the cohomology of $Y_{*}, \widehat{Y}$ and $W^{\dagger}$. The relevant part of this sequence is the exact portion

$$
\begin{aligned}
0 \rightarrow H^{1}\left(Y_{*} ; i \mathbb{R}\right) \rightarrow H^{1}(\widehat{Y} ; i \mathbb{R}) \oplus H^{1}\left(W^{\dagger} ; i \mathbb{R}\right) \stackrel{\Delta}{\longrightarrow} H^{1}\left(T^{2} ; i \mathbb{R}\right) \stackrel{d^{*}}{\longrightarrow} \\
H^{2}\left(Y_{*} ; i \mathbb{R}\right) \rightarrow 0 .
\end{aligned}
$$

We take the convention that the middle map $\Delta(a, b)=i^{*} a-i^{*} b, i$ being the inclusion map. Observe that because $H^{1}\left(Y_{*} ; i \mathbb{R}\right) \otimes H^{2}\left(Y_{*} ; i \mathbb{R}\right)^{*}$ is canonically trivial, an orientation of any two of the three terms $H^{1}(\widehat{Y} ; i \mathbb{R}), H^{1}\left(W^{\dagger} ; i \mathbb{R}\right)$, $H^{1}\left(T^{2} ; i \mathbb{R}\right)$ automatically induces an orientation for the third.

We remind the reader of the definition of a compatibly oriented short exact sequence of oriented finite dimensional vector spaces

$$
0 \longrightarrow V_{0} \stackrel{f_{0}}{\longrightarrow} V_{1} \stackrel{f_{1}}{\longrightarrow} V_{2} \stackrel{f_{2}}{\longrightarrow} V_{3} \stackrel{f_{3}}{\longrightarrow} 0 .
$$


Let $k_{i}=\operatorname{dim} \operatorname{ker} f_{i}$ and $c_{i}=\operatorname{dim} V_{i}-k_{i}$. Then the short exact sequence is compatibly oriented if there is an oriented basis $\left\{v_{i, j} \mid j=1, \ldots, \operatorname{dim} V_{i}\right\}$ for $V_{i}$ such that:

(i) $\left\{v_{i, j} \mid j=1, \ldots, k_{i}\right\}$ is a basis for $\operatorname{ker} f_{i}$, and

(ii) $f_{i}\left(v_{i, k_{i}+j}\right)=v_{i+1, j}$ for $j=1, \ldots, c_{i}=k_{i+1}$.

An orientation of $H^{1}\left(T^{2} ; i \mathbb{R}\right)$ was fixed earlier on as $(i \alpha) \wedge(i \beta)$ and the orientation $i \alpha_{\hat{Y}}$ on $H^{1}(\widehat{Y} ; i \mathbb{R})$ is such that its restriction to $H^{1}\left(T^{2} ; i \mathbb{R}\right)$ is exactly the class $i \alpha$. If $Y_{*}=Y_{1, T}$ or $Y_{T}$ then $H^{1}\left(Y_{*}\right)=H^{2}\left(Y_{*}\right)=0$ and the sequence (9) gives an isomorphism $\Delta: H^{1}(\widehat{Y} ; i \mathbb{R}) \oplus H^{1}\left(W^{\dagger} ; i \mathbb{R}\right) \rightarrow H^{1}\left(T^{2} ; i \mathbb{R}\right)$. This is to be orientation preserving so this induces the orientation $(i \beta)-(i \alpha)$ on $R_{1}\left(\chi_{e}(\widehat{W})\right)$ and the orientation $i \beta$ on $R\left(\chi_{e}(\widehat{W})\right)$.

For the case of $Y_{0, T}$, choose the orientation $i \delta$ for $H^{1}\left(Y_{0, T} ; i \mathbb{R}\right)$ so that $i \delta$ restricts to the class $i \alpha_{\hat{Y}}$. Let $\alpha_{W^{\prime}} \in H^{1}\left(\widehat{W}^{\prime}\right)$ be such that $\Delta\left(\alpha_{\bar{Y}}, \alpha_{W^{\prime}}\right)=0$. Let the induced orientation on $H^{1}\left(\widehat{W}^{\prime} ; i \mathbb{R}\right)$ be represented by $\epsilon i \alpha_{W^{\prime}}, \epsilon= \pm 1$. Then $\Delta\left(0, \epsilon i \alpha_{W^{\prime}}\right)=-\epsilon i \alpha$. On the other hand the span of $i \beta$ under $d^{*}$ of (9) surjects onto $H^{2}\left(Y_{0, T} ; i \mathbb{R}\right)$. Poincare Duality identifies $H^{2}\left(Y_{0, T} ; \mathbb{R}\right)$ with $H^{1}\left(Y_{0, T} ; \mathbb{R}\right)^{*}$. Therefore the orientation induced on $H^{2}\left(Y_{0, T} ; \mathbb{R}\right)$ is given by $\delta^{*}$ such that $\delta^{*}(\delta)>0$. Think of $\beta$ as a differential form on $T^{2}$. Define a differential form supported on the cylindrical neck region of $Y_{0, T}$ by taking $\chi \beta$ where $\chi$ is a bump function depending on the cylindrical parameter. Then $d^{*}(\beta)=d(\chi \beta)$, and $\int_{Y_{0}, T} d(\chi \beta) \wedge \delta>0$, where again we think of $\delta$ as a differential form. Therefore the induced orientation on the span of $i \beta$ is given by $i \beta$ itself. Now we require that $(-\epsilon i \alpha) \wedge(i \beta)$ equals the fixed orientation $(i \alpha) \wedge(i \beta)$ on $H^{1}\left(T^{2} ; i \mathbb{R}\right)$; this forces $\epsilon=-1$. Therefore, $H^{1}\left(\widehat{W}^{\prime} ; i \mathbb{R}\right)$ is oriented according to $-i \alpha$.

\section{References}

[AM] S. Akbulut and J. McCarthy, Casson's invariant for oriented homology 3-spheres, an exposition, Mathematical Notes, 36, Princeton University Press, Princeton, NJ, 1990.

[APS] M.F. Atiyah, V.K. Patodi, and I.M. Singer. Spectral asymmetry and Riemannian geometry I, Math. Proc. Cambridge Philos. Soc. 77 (1975), 43-69.

- Spectral asymmetry and Riemannian geometry II, Math. Proc. Cambridge Philos. Soc. 78 (1975), 405-432.

— Soc. Spectral asymmetry and Riemannian geometry III, Math. Proc. Cambridge Philos. Soc. 79 (1976), 71-99.

[Ch] W. Chen, Casson's invariant and Seiberg-Witten gauge theory, Turkish J. Math. 21 (1997), 61-81.

[CLM] S. Cappell, R. Lee, and E. Miller, Self-adjoint elliptic operators and manifold decompositions I, Comm. Pure Appl. Math. 49 (1996), 825-866.

, Self-adjoint elliptic operators and manifold decompositions II, Comm. Pure Appl. Math. 49 (1996), 869-909.

[L1] Y. Lim, Seiberg-Witten invariants for 3-manifolds in the case $b_{1}=0$ or 1 , to appear Pac. J. Math.

[L2] _ Seiberg-Witten moduli spaces for 3-manifolds with cylindrical-end $T^{2} \times \mathbb{R}^{+}$, submitted for publication. 
[MM] R.R. Mazzeo and R.B. Melrose, Analytic surgery and the eta invariant, Geom. Funct. Anal. 5 (1995), 14-75.

[MT] G. Meng and C. Taubes, $\underline{S W}=$ Milnor Torsion, Math. Res. Lett. 3 (1996), 661-674.

[MR] R. Meyerhoff and D. Ruberman, Cutting and pasting and the $\eta$-invariant, Duke Math. J. 61 (1990), 747-761.

[MOY] T. Mrowka, P. Ozsváth, and B. Yu, Seiberg-Witten monopoles on Seifert fibred spaces, Comm. Anal. Geom. 5 (1997), 685-791.

Department of Mathematics, University of California at Santa Barbara, Santa BARBARA, CA 93106

E-mail address: ylim@gauss.math.ucsb.edu 